\title{
Avo Investigations Of Shallow Marine Sediments
}

\author{
M. Riedel ${ }^{1}$ and F. Theilen ${ }^{2}$ \\ ${ }^{1}$ School of Earth and Ocean Science, University of Victoria, B.C. Canada \\ 2 Institute of Geosciences, Department of Geophysics, University Kiel, Germany
}

AVO/AVA (Amplitude Versus Offset/Angle) analysis has been used to determine the physical characteristics of shallow marine sediments. The parameter that can be constrained, are P- and S- wave velocity, bulk density and acoustic attenuation. Shear modulus and other geotechnical properties such as grain size can be inferred from these parameters. The theory of AVO investigations is given by the Zoeppritz equations (Zoeppritz, 1919), which allow the computation of the reflection and transmission coefficients as a function of angle of incidence. AVO has been widely used in the petroleum industry, usually for frequencies of $20-100 \mathrm{~Hz}$. High frequency shallow sediment AVO studies require special analysis including careful geometry and source and receiver directivity corrections. In the past, marine sediments have been modeled as elastic materials. However, viscoelastic models with absorption are more realistic. At large angle of incidence, AVA-functions derived from viscoelastic models differ significantly from those with purely elastic properties. The influence of S-wave velocity on the reflection coefficient is rather small (especially for the low S-wave velocities encountered at the seafloor). On the other hand, P-wave velocity and density show a considerably stronger effect (Fig. 1). Thus, it will be difficult, or nearly impossible, to extract the S-wave parameter from AVA trends, especially when the signal-to-noise ratio is low.

In order to measure the reflection coefficient in a seismogram, the peak amplitudes of the direct wave and the seafloor reflection in a CMP (Common Mid Point) gather are determined and corrected for spherical divergence, and source and receiver directivity (Fig. 2). At CMP locations showing different AVA characteristics, the sediment parameters $\mathrm{P}$ - and $\mathrm{S}$-wave velocity, density and absorption are determined by using a Sequential Quadratic Programming (SQP) inversion technique. The use of the viscoelastic model within the inversion gives better results in fitting the data than the elastic model without absorption.

The introduction of constraints, e.g. empirical relationships given by core investigations, can improve and stabilize the inversion results.

\begin{tabular}{|c|c|c|}
\hline Parameter & CMP 570 & CMP 4000 \\
\hline P-wave $[\mathrm{m} / \mathrm{s}]$ & 1453 & 1622 \\
\hline S-wave $[\mathrm{m} / \mathrm{s}]$ & 92 & 358 \\
\hline density $\left[\mathrm{kg} / \mathrm{m}^{3}\right]$ & 1220 & 1380 \\
\hline Q P-wave & 14 & 787 \\
\hline Q S-wave & 9 & 25 \\
\hline
\end{tabular}

Table 1. Inverted sediment properties of CMP-Bin 570 and CMPBin 4000 .
In Fig. 2, a soft mud layer, with a typical low P-wave velocity close to water velocity, forms the seafloor at CMP-Bin 570 whereas at CMP-Bin 4000 the seafloor contains boulder clay, a mixture of sand and gravel with a higher P-wave velocity and density.

In both cases the inversion gives reasonable results for the first three sediment parameters. Note that the value of the S-wave velocities have an uncertainty of more than $100 \%$ due to the insensitivity of the reflection coefficient to this parameter.

The investigation of deeper sediment layers by AVA requires a good estimate of the absorption (described by the Q-factor) within the first sediment layer.

The influence of $\mathrm{Q}$ on the reflection coefficient is enhanced in the wide-angle domain. However, our measurements were restricted to a maximum angle of incidence of $60^{\circ}$, which results in relatively high uncertainties in the inversion result with respect to the absorption factor Q. Further investigations on the absorption in shallow marine sediments, e.g. by spectral division methods of broadband multichannel seismic data, must be carried out in the future.

A posteriori probability distributions for linearized inversion and non-linear approaches (simulated annealing and Gibbs sampling) are subject of ongoing research.

\section{REFERENCES}

Zoeppritz, R., 1919. On the reflection and propagation of seismic waves, Erdbebenwellen VIIIB, Göttinger Nachrichten, I, 66-84 

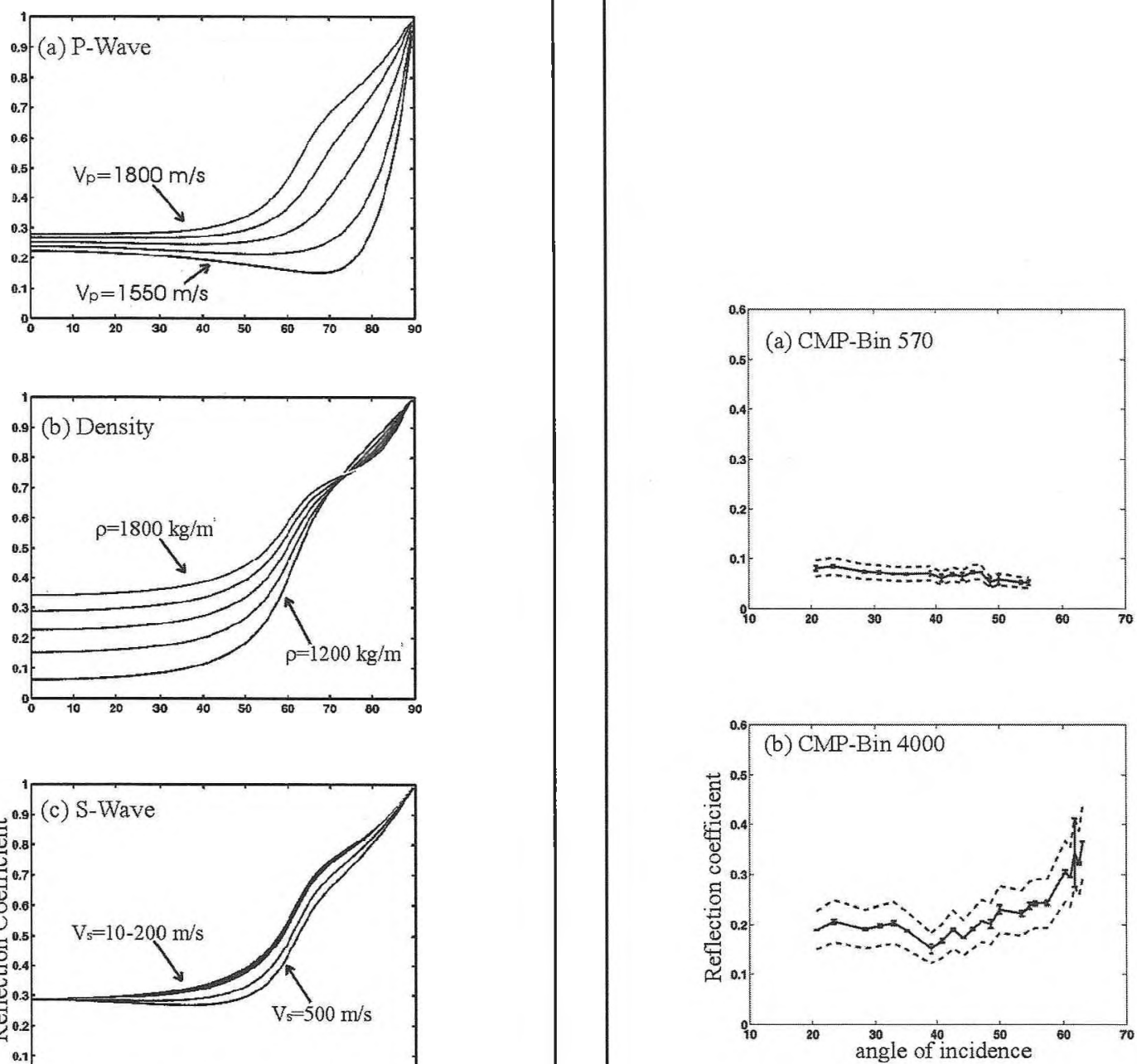

Fig. 1. Influence of (a) P-wave velocity, (b) density, and (c) Swave velocity on the reflection coefficient as a function of angle of incidence. The parameters of the viscoelastic sediment model are: $\mathrm{P}$-wave velocity $=1700 \mathrm{~m} / \mathrm{s}$, S-wave velocity $=100 \mathrm{~m} / \mathrm{s}$, density $=1300 \mathrm{~kg} / \mathrm{m} 3$, and $\mathrm{Qp}=\mathrm{Qs}=10$.

Fig. 2. Calculated reflection coefficient at (a) CMP-Bin 570 (mud), and (b) CMP-bin 4000 (boulder clay). Error bars indicate variability within each bin. Lower and upper bounds for the reflection coefficient have been included based on an uncertainty in the source directivity of $20 \%$. 